\title{
Assessment of elbow functional outcome after closed reduction and percutaneous pinning of displaced supracondylar humerus fractures in children
}

\author{
Rutarama $\mathrm{A}^{1}{ }^{\mathbb{0}}$, Firth $\mathrm{GB}^{2} \mathbb{B}$ \\ 1 MBBCh; Registrar* \\ 2 MBBCh, FCS(Orth), MMed(Orth); Consultant* \\ *Department of Orthopaedic Surgery, University of the Witwatersrand, Chris Hani Baragwanath Academic Hospital, Johannesburg, South Africa
}

Corresponding author: $\operatorname{Dr}$ GB Firth, Faculty of Health Sciences, Orthopaedic Surgery Division, University of the Witwatersrand, Private Bag 3 , Wits 2050, South Africa; tel: +27 (11) 717 2538; email: greg.firth@gmail.com

\begin{abstract}
Background: The aim of the current study was to establish the functional outcome of Gartland grade III extension-type supracondylar fractures at 24 weeks after closed reduction and percutaneous pinning (CRPP) without physiotherapy. The objectives of this study were 1) to measure the elbow range of motion (ROM) at three, six, 12 and 24 weeks after CRPP for grade III supracondylar fractures; 2 ) to assess the functional outcome of the injured limb using the Paediatric Outcome Data Collection Instrument (PODCI) score; 3 ) to compare elbow functional outcome of children less than 7 years old versus children more than 7 years old; and 4) to assess other risk factors or complications associated with poor outcomes after CRPP (including age and associated soft tissue injury).

Patients and methods: A prospective cohort study was performed. The study included 38 children under the age of 14 years with grade III extension-type supracondylar fractures who had manipulation under anaesthesia (MUA) and crossed K-wire fixation. ROM of the affected elbow (flexion, extension, pronation and supination) was measured at three, six, 12 and 24 weeks after CRPP by the same author (AR) at each visit. The unaffected elbow was used as a control. The PODCI was also recorded. No physiotherapy was prescribed.

Results: Thirty-eight children were included in the study. All components of elbow ROM improved at 24 weeks $(p<0.001)$. At 12 but not 24 weeks, the mean elbow extension was reduced in comparison with the controls $(p=0.009)$. Patients less than 7 years of age recovered extension more rapidly $(p=0.001)$. Seventy-six per cent of the children achieved satisfactory PODCI results at the 24 -week final follow-up. Nerve palsy (18.4\%) and severe soft tissue injuries (7.9\%) were the main contributory factors to a poor outcome on the $\mathrm{PODCl}$ assessment.

Conclusion: The majority of children with displaced supracondylar fractures recover full ROM after CRPP by 24 weeks without physiotherapy. Older children, or those with associated neurovascular and soft tissue injuries had poor functional outcomes. Further studies are needed to assess if these patients will benefit from physiotherapy.
\end{abstract}

Level of evidence: Level 4

Keywords: child, Gartland grade III supracondylar fracture, outcomes, closed reduction percutaneous K wires

Citation: Rutarama A, Firth GB. Assessment of elbow functional outcome after closed reduction and percutaneous pinning of displaced supracondylar humerus fractures in children. SA Orthop J 2019;18(4):14-19. http://dx.doi.org/10.17159/2309-8309/2019/v18n4a1

Editor: Prof J du Toit, Stellenbosch University, Cape Town, South Africa

Received: January 2019

Accepted: May 2019

Published: November 2019

Copyright: @ 2019 Rutarama A, Firth GB. This is an open-access article distributed under the terms of the Creative Commons Attribution Licence, which permits unrestricted use, distribution and reproduction in any medium, provided the original author and source are credited.

Funding: This study did not require any funding.

Conflict of interest: Both authors declare having no conflict of interest with regard to this study. 


\section{Introduction}

Closed reduction and percutaneous pinning (CRPP) of Gartland grade III supracondylar fractures in children has become the gold standard of care in the last 50 years in the global orthopaedic literature..$^{1-4}$ This is known as the modern concept of skeletal stabilisation and soft tissue management in paediatric orthopaedic surgery. Authors have cautioned against the open operative management of these fractures, and this has resulted in improved functional outcomes. ${ }^{4}$ Many authors have also cautioned against physiotherapy in the management of uncomplicated children's fractures. ${ }^{4}$ Even though there may be a delay in recovery of elbow range of motion (ROM), outcomes at one year have been shown to be equivalent (with or without physiotherapy) for Gartland grade III supracondylar fractures. ${ }^{5}$ There is paucity in the literature about the functional outcome after Gartland grade III supracondylar humerus fractures. Pressure from parents and patients for early intervention and early rehabilitation for rapid and complete return to activity are an ever-increasing demand in today's modern lifestyle. More recent evidence for physiotherapy after CRPP for supracondylar fractures in children found no difference one year post treatment. ${ }^{6}$ Keppler et al. showed a small benefit of physiotherapy at six weeks and no benefit at one year but Schmale et al. showed no benefit at all.5,6

The outcome of grade III supracondylar fractures in South Africa has not been studied prospectively before. The aim of the current study was to establish the functional outcome of Gartland grade III extension-type supracondylar fractures at 24 weeks after CRPP without physiotherapy. The objectives of this study were 1) to measure the elbow ROM at three, six, 12 and 24 weeks after CRPP for grade III supracondylar fractures; 2) to assess the functional outcome of the injured limb using the Paediatric Outcome Data Collection Instrument (PODCl) score; 3) to compare elbow functional outcome of children less than 7 years old versus children more than 7 years old; and 4) to assess other risk factors or complications associated with poor outcomes after CRPP.

\section{Materials and methods}

Inclusion criteria included children under the age of 14 years with a unilateral extension-type grade III supracondylar fracture requiring CRPP. Exclusion criteria included the presence of associated fractures on the ipsilateral or contralateral upper limb, children requiring open reduction, and children lost to follow-up.

All children were treated with the following protocol: analgesia followed by a backslab in a comfortable position, usually at $90^{\circ}$ of flexion. The child was taken to theatre as soon as possible - ideally the same night - for closed reduction and percutaneous cross $\mathrm{K}$ wires. The lateral wire was inserted first with the elbow flexed and then, to reduce the risk of ulnar nerve injury, the elbow was extended and the medial $\mathrm{K}$ wire inserted (Figure 1).

Considering age as a risk factor for poor return of elbow extension, we compared two groups. The first group of children were younger than 7 years and the second group of children were aged 7 years or older.

No physiotherapy was prescribed as standard practice. Children were encouraged to return to normal activities as soon as the $\mathrm{K}$ wires were removed at three weeks, guided by the residual elbow pain.

All associated soft tissue injuries and complications were assessed and recorded at each visit. Control X-rays were taken at six weeks after CRPP for every patient and at 24 weeks for those who still had residual impaired ROM or any clinical suspicion of malunion (Figure 1)

At three weeks, ROM measurements of the normal elbow were done with a goniometer (flexion, extension, pronation and supination) by the first author (AR) for the control; and baseline data on the affected elbow was also recorded. The ROM on the affected side was then further recorded at six, 12 and 24 weeks.

The PODCl is a questionnaire developed by the Paediatric Orthopaedic Society of North America (POSNA) to measure functional outcomes after an orthopaedic intervention in children. It was validated and used in a wide array of musculoskeletal conditions including congenital, metabolic and traumatic. The versatility of the $\mathrm{PODCl}$ in using certain applicable parts makes it easier to use and more appropriate than just relying on physical findings. The use of PODCl after paediatric orthopaedic trauma is a good tool in assessing the function of these children. The current study used two of its five major components relevant to the upper limb: upper limb physical function (assesses basic activities of daily living such as combing hair, buttoning a shirt, eating with a spoon and carrying heavy books); and a general happiness score about the outcome of the intervention. The PODCl questionnaire was used at the final follow-up to look, in particular, at the patient's upper extremity functional outcome scores as assessed by the

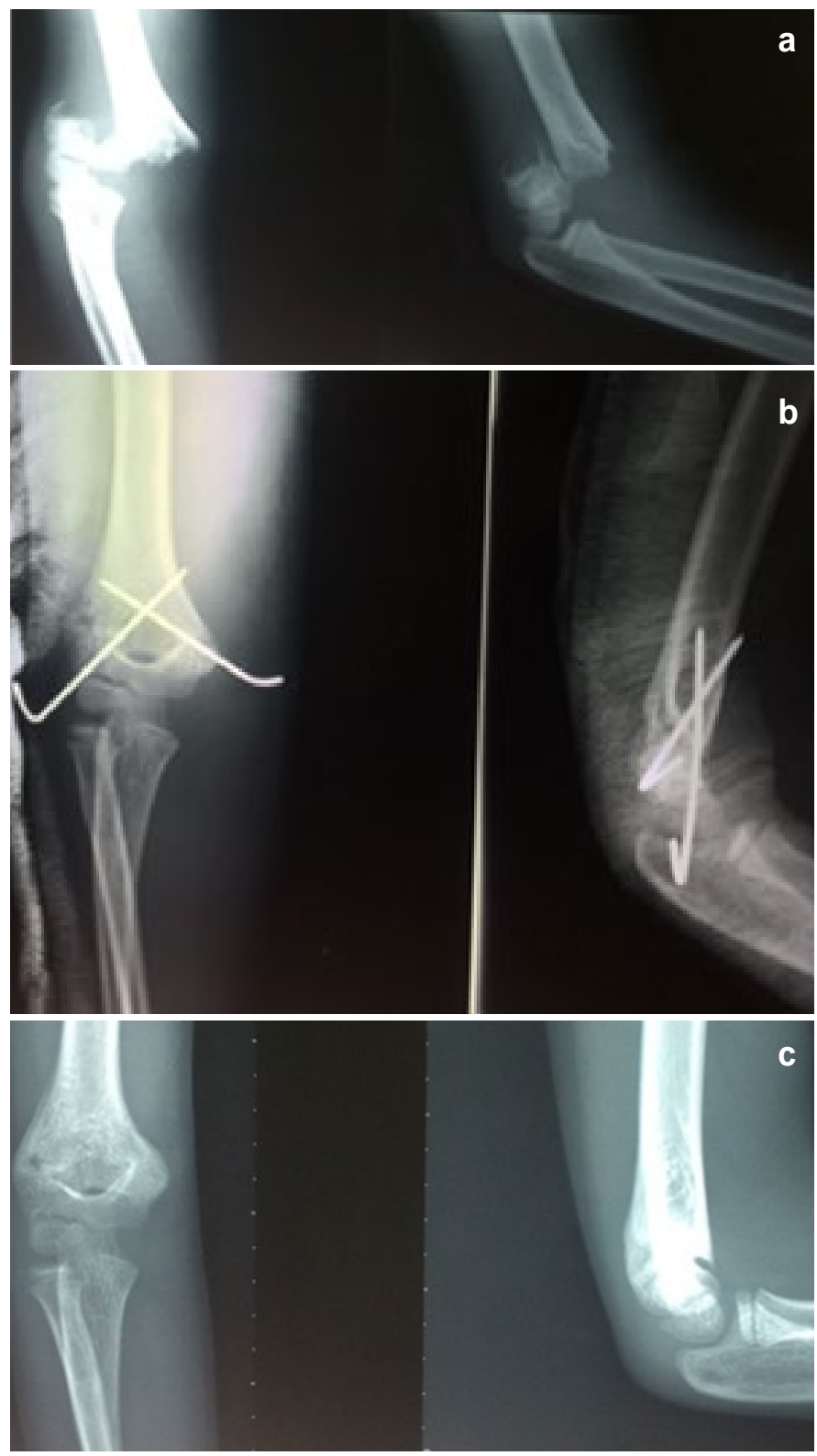

Figure 1. a) Demonstration of the extension type Gartland grade III supracondylar fracture, b) CRPP with cross pinning, and c) outcome at 24 weeks after CRPP 
parent, and the parent's happiness about their child's outcome after CRPP. This evaluation aimed at gaining an understanding of clinical outcomes and perceptions from patients or parents about the outcome. Scores for each of the answers were then calculated in terms of a percentage to assess how many were able or not able to perform basic activities of daily living as a result of the injury and its management.

In the PODCl grading system for upper limb function, the parent/ caregiver reports as to whether their child could perform specific activities easily with no assistance (easy); with some difficulty but still able to perform them (little hard); and almost unable to perform task or performs it incompletely (very hard).

Overall outcome for the PODCl was assessed by the parent/ caregiver as either satisfactory (very or somewhat) or dissatisfactory (very or somewhat).

Raw data was entered into an Excel spreadsheet, and statistical software (Stata) was used to analyse the data. Using the skewness kurtosis test for the normality of data, some of the data was not

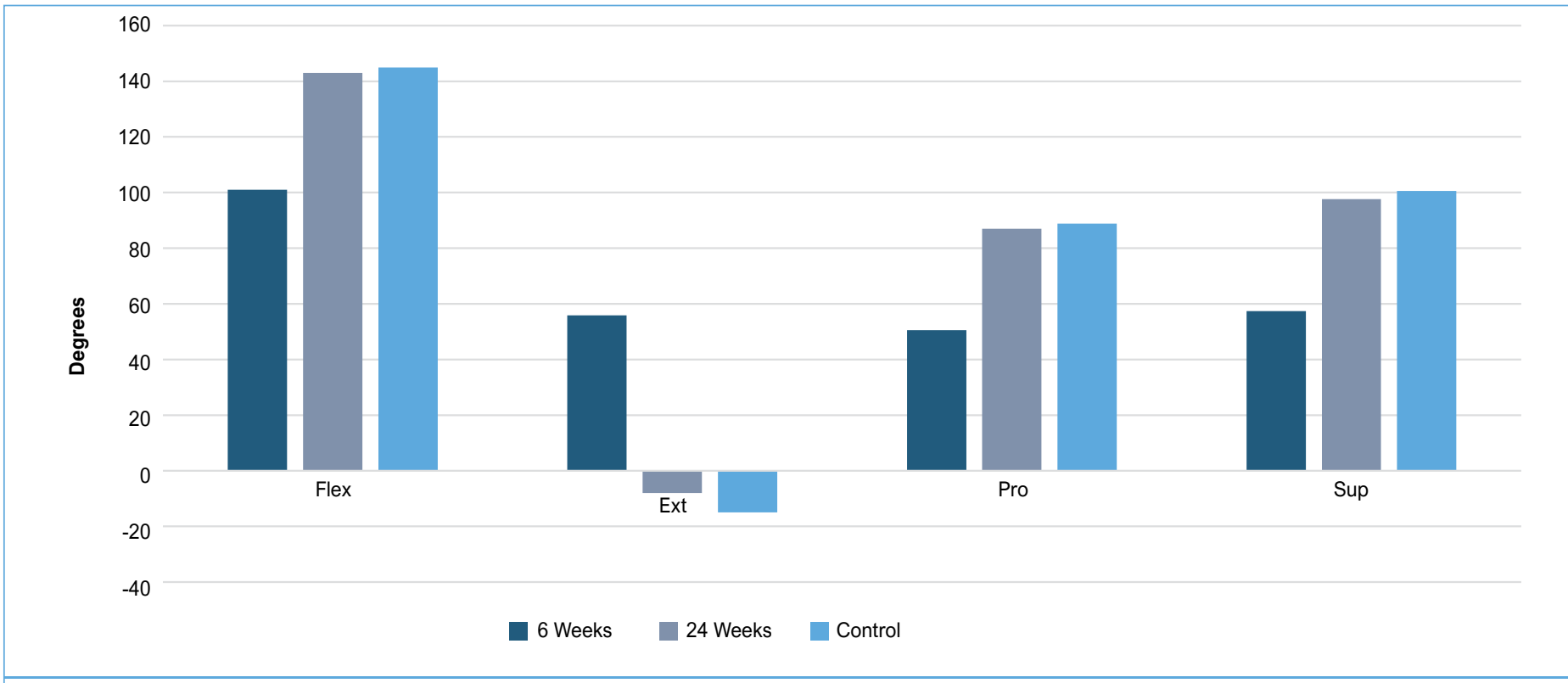

A. Flexion

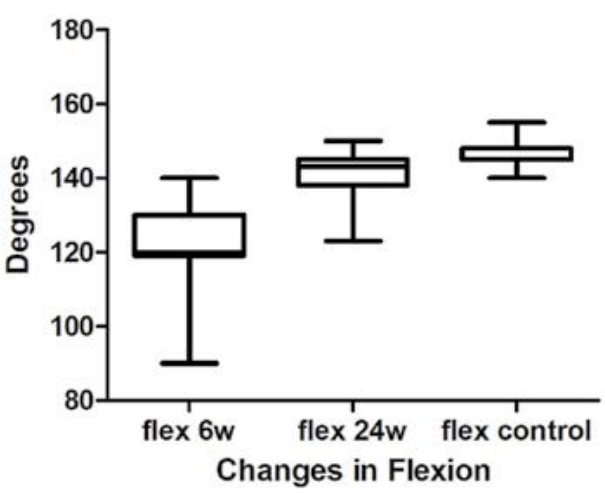

C. Pronation

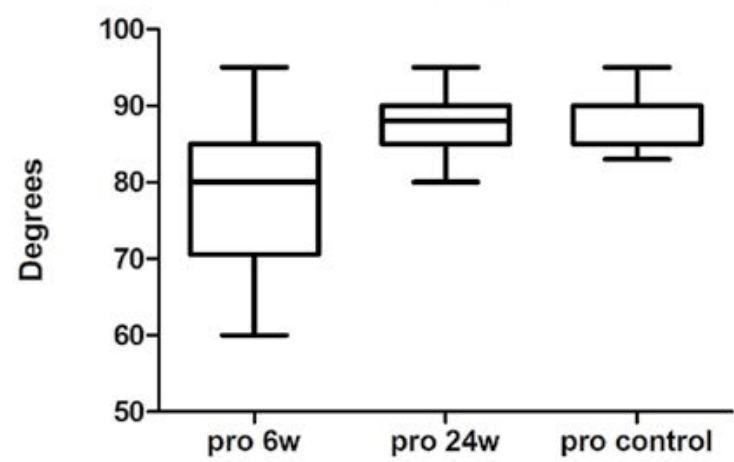

B. Extension

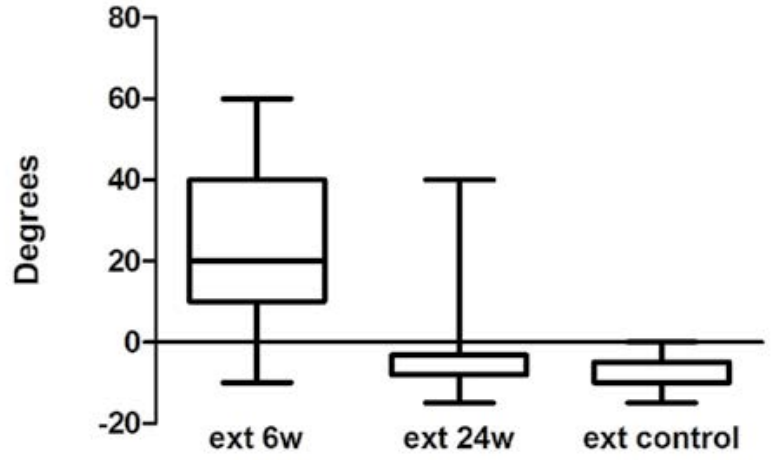

D. Supination

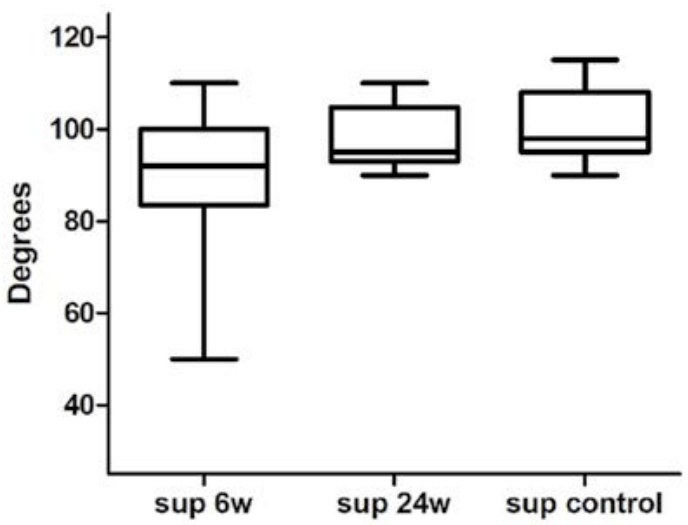


Table I: Summary comparing ROM at final follow-up with presence or absence of complications

\begin{tabular}{|l|c|c|c|}
\hline \multicolumn{1}{|c|}{ Final follow-up } & Median flexion (IQR) & Median extension (IQR) & Median pronation (IQR) \\
\hline No complications $(n=26)$ & $145^{\circ}\left(3^{\circ}\right)$ & $-8^{\circ}\left(7^{\circ}\right)$ & $90^{\circ}\left(2^{\circ}\right)$ \\
\hline Complications $(n=12)$ & $143^{\circ}\left(6^{\circ}\right)$ & $-6^{\circ}\left(8^{\circ}\right)$ & $94^{\circ}\left(8^{\circ}\right)$ \\
\hline
\end{tabular}

IQR: inter-quartile range

Table II: Summary of paediatric outcome data collection instrument for upper limb function of the children as assessed by the parent

\begin{tabular}{|c|c|c|}
\hline Easy $(n=25,66 \%)$ & Little hard $(n=6,16 \%)$ & Very hard $(n=7,18 \%)$ \\
\hline \multirow[t]{4}{*}{ No complications } & 4 ulnar nerve palsies & 2 open fractures \\
\hline & 2 over 7 years of age & 2 ulnar nerve palsies \\
\hline & & 1 radial nerve palsy \\
\hline & & 2 over 10 years of age \\
\hline
\end{tabular}

Parents reported whether their child could perform specific activities easily with no assistance (easy), with some difficulty but still able to perform them (a little hard) and almost unable to perform task or performs it incompletely (very hard).

Table III: Summary of paediatric outcome data collection instrument for parental satisfaction of their child's outcome

\begin{tabular}{|c|c|c|c|c|}
\hline Very satisfied & Somewhat satisfied & Neutral & Somewhat dissatisfied & Very dissatisfied \\
\hline $25(66 \%)$ & $4(11 \%)$ & $3(8 \%)$ & $3(8 \%)$
\end{tabular}

normally distributed and as a result, non-parametric data analysis was used. Epidemiological data such as age, sex, affected upper extremity and PODCl were reported descriptively. ROM measurements on the affected limb were compared with control measurements at six and 24 weeks. Wilcoxon and Mann-Whitney $\mathrm{U}$ tests were used for the data that was skewed and compared with two age groups - those older than or equal to 7 years and those under 7 years of age. Results were represented in ordinal tables as well as box plots and whisker tables and interpreted to assess the hypothesis. Quantitative data of soft tissue injuries such as nerve and vascular injuries, compartment syndrome, pin-tract sepsis and PODCl scores were also reported descriptively.

Signed informed consent was completed by all parents and signed informed assent was obtained from all children who could understand the request. Ethics approval was obtained from the University Human Research Ethics Committee.

\section{Results}

This study was a prospective cohort study and included 53 children. Fifteen children were lost to follow-up, leaving 38 children eligible for inclusion in the study. Thirty out of the 38 children completed all four follow-up visits. Six missed one and two missed two followup visits. The mean age at presentation was 7.5 years (SD 2.5). Twenty-five (66\%) patients were male and 29 (76\%) patients injured the left side.

\section{Range of movement}

Twenty-nine (76\%) of the children gained $90 \%$ of normal elbow $\mathrm{ROM}$ at 24 weeks. A significant improvement in the elbow ROM was noted between six and 24 weeks with a p-value of $<0.001$ using Wilcoxon paired tests (Figures 2a-d).

Elbow extension improved less compared to the other ranges in the elbow and forearm movement during the first 12 weeks. In addition, a statistically significant difference in loss of elbow extension was present in children 7 years of age or older. This was noted at six and 12 weeks with p-values of 0.001 and 0.009 respectively using the Mann-Whitney $U$ test. At 24 weeks this difference was still observed although not statistically significant.

There was no statistical difference in the ROM at the final followup whether the patients had a complication or not (Table I).

\section{Outcomes}

The PODCl assessed the function of the child as reported by the parent/caregiver at final follow-up as well as the overall parent satisfaction regarding the child's outcome. Regarding the $\mathrm{PODCl}$ score of the upper limb physical function at final follow-up, 25 (66\%) of the children easily performed basic functional activities; six (16\%) found it a little hard and seven $(18 \%)$ found it very hard and could not perform them at all. In the six patients who found it a little hard, four of them had sustained an iatrogenic ulnar nerve injury and two others were older than 7 years of age. The seven children who found it very hard to perform basic activities of daily living included two open injuries, two ulnar nerve injuries, one radial nerve injury (sustained at the time of the fracture), and two were older than 10 years of age (Table II). Twenty-nine (76\%) in the cohort attained $90 \%$ of the normal elbow ROM at 24 weeks, but only 25 (66\%) of the children could easily perform basic upper limb physical functional activities with parents being very satisfied about the outcome (Table III).

In terms of PODCl scores that assessed happiness of the parent/caregiver about the outcome condition, 29 parents $(77 \%)$ were either very or somewhat satisfied about the outcome of their child's condition. Six (16\%) of the parents were somewhat or very dissatisfied about the outcome of their child's condition at final follow-up (Table III). All six of the children whose parents were either somewhat or very dissatisfied had a reason for this - it was noted that two of these children had sustained open supracondylar fractures: one developed compartment syndrome, two missed two follow-up visits and one was 13 years old. Four out of seven cases with neuropraxia had residual elbow dysfunction at 24 weeks and their parents were dissatisfied.

\section{Associated injuries/complications}

All children with associated injuries $(n=12)$ were included in the final outcome at 24 weeks but showed no statistical difference in the median ROM when compared with children who had no complications $(n=26)$ (Table I). There were two cases with open supracondylar fractures in the study and both resulted in elbow fixed flexion deformities of more than $30^{\circ}$ at the 24-week final follow-up. 
Associated soft tissue injuries included seven neuropraxias (18\%) (six ulnar and one radial nerve). Four of the six ulnar nerve injuries were iatrogenic and two were associated with the fracture pre-operatively. The seven children with nerve injuries had a mean age of 10 years. All but one of these nerve injuries progressively improved and fully recovered by the twelfth week.

The child who developed compartment syndrome had a very slow and incomplete recovery of the radial nerve at the 24-week final follow-up. Fasciotomy was done urgently after the CRPP but resulted in a stiff elbow due to Volkmann's ischaemic contracture with an FFD of $38^{\circ}$ by the 24-week follow-up. Ulnar and radial nerve function was still affected by the end of the 24-week follow-up with nerve conductive studies indicating the injury to be a neuropraxia.

\section{Discussion}

The current cohort (38 children) of grade III supracondylar fractures was larger than other prospective studies noted in the literature..$^{5-8}$ In the current study the male:female ratio was $1.5: 1$, half the $3: 1$ global figure given for these fractures. The current population has marginalised gender disparities in terms of activities performed by both sexes which differs from other studies on supracondylar fractures. ${ }^{4,7-14}$ In the current study, 29 (76\%) had left-sided supracondylar fractures, in keeping with global literature in which the left side was predominantly affected. ${ }^{4-10,13-15}$

Spencer et al. showed that there is rapid improvement in elbow ROM in the first four weeks after removal of the cast followed by a slower increase in ROM for up to one year. In their study, recovery of ROM was slower in children older than five years and in those with severe injury patterns. They confirmed that recovery of full extension was slower in fractures with severe associated injuries around the elbow. ${ }^{7}$ This concurs with the current study results in which older children (over the age of 7 years) and those with associated injuries took longer to regain full ROM but at the 24-week final follow-up had similar ROM to those without complications. Spencer et al.'s cohort achieved 95\% of the normal elbow ROM by 24 weeks. Comparable results were noted by Zionts et al. where $94 \%$ gained normal elbow ROM at 26 weeks and $98 \%$ at 52 weeks after CRPP. 13

The PODCI has been used to measure outcome in children after many orthopaedic interventions. ${ }^{16}$ Wang et al. also used PODCI to assess the functional outcome of Gartland grade III supracondylar fractures with early neurovascular compromise in children and showed it to have a high sensitivity and specificity compared with other outcome measures. ${ }^{11}$ These papers highlight that anatomical function (including anatomical reduction, alignment and full functional arc) does not always equate with good clinical outcome (such as pain scores and the ability to perform a task) - the 12 children $(31 \%)$ in the current study who did not show satisfactory $\mathrm{PODCl}$ results either sustained associated soft tissue injuries (nerve injury, open fractures or compartment syndrome) or were older than 7 years of age, despite near normal ROM at final follow-up (Table II). The benefit of PODCl is that it can add further information to the final assessment and outcome of these children by assessing more than just the ROM and anatomical reduction (Tables II and III). In the current study, the PODCI proved to be more sensitive to musculoskeletal changes after CRPP than physical examination alone. Lerman et al. observed that PODCl is an efficient instrument in the assessment of function after an orthopaedic intervention. ${ }^{16}$ The current study supports the findings of Lerman et al. because restoration of anatomy does not always equate to function. Up to a third of patients with full ROM at the 24-week final follow-up could not perform basic upper limb physical functional activities easily and parents were not very satisfied about the outcome.

In the current study, we have shown that supracondylar fractures can be a source of physical disability in those children with complications. Children who sustained nerve injuries, open fractures, compartment syndrome, and those with age greater than 7 years had reduced elbow ROM and PODCl results at the 24-week follow-up. Spencer et al. showed similarly that in patients who were older than 5 years of age, the relative arc of motion was decreased by $3-9 \%$ compared with patients younger than 5 years of age. ${ }^{7}$ In the same study, it was noted that the more severe the injury, the slower the elbow motion recovery. This correlated with the findings of the current study where the children who sustained open fractures, compartment syndrome or nerve injuries had relatively poor elbow functional outcome at the 24-week follow-up.

Complications after supracondylar humerus fractures are not uncommon. Acutely these include open soft tissue injuries, neurovascular injuries and compartment syndrome. Some studies have shown incidence of vascular injuries of up to $20 \% .4,8,11,14,17$ Badkoobehi et al. noted that $20 \%$ of displaced supracondylar fractures have an associated vascular injury (ranging from vessel spasm to overt vessel damage). ${ }^{17}$ In the current study, only the case with compartment syndrome had vascular compromise due to spasm and kinking by the fracture fragment displacement compounded by subsequent compartment syndrome - flow was re-established after CRPP and fasciotomy.

In terms of nerve injuries associated with extension-type supracondylar fractures, the most commonly injured nerve is the radial nerve followed by the median nerve and ulnar nerve.,3,14 In the current study the most commonly injured nerve was the ulnar nerve (excluding iatrogenic injuries) and then the radial nerve. The ulnar nerve injury is one of the most common iatrogenic complications recorded when using the crossed-pinning technique. Pre-operative nerve injuries occur most commonly in older children.3,4,14 We observed an overall incidence of $18.4 \%$ (seven children) of nerve injuries and the mean age for these children was 10 years, which confirmed the findings in the literature. Other studies in the literature have reported results within the same range.3,4,11,14 The children with associated nerve injuries all had reduced ROM (especially extension) at final follow-up $(p=0.009)$. In our study, there were six ulnar (16\%) and one radial nerve injuries. Four (10.5\%) of the ulnar nerve injuries were iatrogenic, which was higher than other studies such as the one conducted by Prashant et al. (6.5\%). ${ }^{3}$ Ongoing training of junior staff is essential but not the focus of this study a safer alternative for junior staff may be two lateral $\mathrm{K}$ wires. The majority of patients who sustained an associated nerve injury/ open fracture were in the older group (over 7 years) and had poor outcomes compared with those without these associated injuries.

One case developed compartment syndrome after CRPP. Fasciotomy was done urgently after CRPP with subsequent Volkmann's ischaemic contracture, ulnar nerve neuropraxia and a fixed flexion deformity of $38^{\circ}$ at 24 weeks. Robertson et al. showed that neurovascular injuries and older age were both risk factors for developing compartment syndrome in children with grade III supracondylar fractures, as in this case. ${ }^{18}$

Sinikumpu et al. performed a population-based long-term followup study of 81 children with Gartland I-III fractures; of these, 25 were Gartland III fractures and they found that only $76 \%$ of these had a satisfactory outcome after closed or open reduction and percutaneous pinning according to Flynn's criteria. ${ }^{19}$ The current study was looking only at Gartland III fractures (38 cases) and also found that although most patients at 24 weeks had return of full ROM, they had reduced scores using the PODCl in over $30 \%$ of cases. This and the current study highlight that these fractures often have mild symptoms and deformity at final follow-up, despite accurate reduction and fixation. Both studies found that results were worse in older patients - Sinikumpu et al. in children older than 10 years of age and the current study in children older than 7 years of age. ${ }^{19}$ 
Tumomilehto et al. reviewed 264 children with Gartland III fractures and found that despite unsatisfactory pin fixation in a third of cases, significant malunion was rare at long-term follow-up. ${ }^{20}$

Controversies exist on the effectiveness of physiotherapy in supracondylar fractures after CRPP. Sub-optimal limb function after an orthopaedic intervention due to poor or delayed rehabilitation may raise medico-legal issues. Keppler et al. ${ }^{6}$ randomised two groups of children with supracondylar fractures (Gartland grades II and III) for physiotherapy (21 children) and the other group without physiotherapy, looking at the effectiveness of physiotherapy in these children after open reduction and internal fixation. At 12 weeks they noticed a better return of elbow ROM in the physiotherapy group but at one year the groups were the same. Unlike the current study, none of these children had neurological injuries and they all had an open reduction which may have predisposed them to higher rates of elbow stiffness. If the neurological injuries were to be excluded from the current series, the results would be very similar. In contrast, Schmale et al. ${ }^{5}$ demonstrated that there was no benefit of physiotherapy to children $(n=61)$ with Gartland grades I, II and III supracondylar fractures, managed by either casting or CRPP. The inclusion of grades I-III could have skewed the benefits of physiotherapy to a small subpopulation of this cohort with displaced supracondylar fractures. Larger multi-centre randomised controlled trials (RCTs) on the role of physiotherapy in displaced supracondylar fractures are needed.

To our knowledge, this is the first prospective study in South Africa focusing on the functional outcome of extension-type Gartland grade III supracondylar humerus fractures assessing both elbow ROM and PODCl after CRPP.

Although this study was prospective, there was a short period of data collection (24 weeks) and limited sample size. There was a large loss of patients $(n=15,26 \%)$ to follow-up due to loss or change of contact details, and the PODCl questionnaire was limited to only two items that were relevant to the study of the upper limb.

\section{Conclusion}

This study has demonstrated that most children with Gartland grade III extension-type supracondylar fractures gain full elbow ROM and have good functional outcome by 24 weeks after closed reduction and percutaneous cross $\mathrm{K}$ wires. Further findings demonstrated that functional outcome does not necessarily equate to good or excellent clinical outcome with the use of the PODCI tool, especially in those children who were older than 7 years of age and those that sustained severe soft tissue injuries (open fractures, nerve injuries, compartment syndrome or internal degloving injuries). The value of a protocol-driven rehabilitation programme which may include physiotherapy for patients identified to be at risk of poor outcome (those over 7 years of age or with associated soft tissue injuries) requires further prospective study.

\section{Ethics statement}

This study was approved by the Human Research Ethics Committee of the University of Witwatersrand (clearance no. M150901) and consent was obtained from the CEO of Chris Hani Baragwanath Academic Hospital.

Signed informed consent was completed by all parents and signed informed assent was obtained from all children who could understand the request.

\section{Declarations}

The authors declare authorship of this article and that they have followed sound scientific research practice. This research is original and does not transgress plagiarism policies.

\section{Author contributions}

AR conceived of the idea together with GBF and was the primary data collector in all cases. AR assisted with application to the departmental research committee and ethics board and wrote the initial manuscript.
GBF conceived of the research idea and helped to develop the study protocol. GBF contributed to writing up the manuscript for submission and helped with revisions.

\section{ORCID}

A Rutarama (D) https://orcid.org/0000-0002-8354-3371

GB Firth (D) https://orcid.org/0000-0002-1594-2290

\section{References}

1. Zionts LE, McKellop HA, Hathaway R. Torsional strength of pin configurations used to fix supracondylar fractures of the humerus in children. J Bone Joint Surg Am. 1994 Feb;76(2):253-56.

2. Howard A, Mulpuri K, Abel MF et al. The treatment of pediatric supracondylar humerus fractures. J Am Acad Orthop Surg. 2012 May;20(5):320-27. doi: 10.5435/JAAOS-20-05-320.

3. Prashant $\mathrm{K}$, Lakhotia $\mathrm{D}$, Bhattacharyya TD et al. A comparative study of two percutaneous pinning techniques (lateral vs mediallateral) for Gartland type III pediatric supracondylar fracture of the humerus. J Orthop Traumatol. 2016 Sep;17(3):223-29. doi: 10.1007/s10195-016-0410-2.

4. Skaggs D. Rockwood and Wilkins Fractures in Children, 7th Edition. Volume 3. Beaty JH, Kasser JR ed. Philadelphia: Lippincott Williams \& Wilkins; 2010.

5. Schmale GA, Mazor S, Mercer LD et al. Lack of benefit of physical therapy on function following supracondylar humeral fracture: A randomized controlled trial. J J Bone Joint Surg Am. 2014 Jun 4;96(11):944-50.

6. Keppler P, Salem K, Schwarting B et al. The effectiveness of physiotherapy after operative treatment of supracondylar humeral fractures in children. J Pediatr Orthop. 2005 May-Jun;25(3):314-16.

7. Spencer HT, Wong M, Fong YJ et al. Prospective longitudinal evaluation of elbow motion following pediatric supracondylar humeral fractures. J Bone Joint Surg Am. 2010 Apr;92(4):904-10. doi: 10.2106/JBJS.I.00736.

8. Anvekar PM, Nimbargi SS, Akshay MK et al. A prospective study of surgical management of the displaced supracondylar fractures of humerus in children with k wire fixation. IJOS 2017;3(3):176-81.

9. Houshian S, Mehdi B, Larsen MS. The epidemiology of elbow fracture in children: Analysis of 355 fractures, with special reference to supracondylar humerus fractures. J Orthop Sci. 2001;6(4):312-15.

10. Abzug JM, Herman MJ. Management of supracondylar humerus fractures in children: Current concepts. J Am Acad Orthop Surg. 2012 Feb;20(2):69-77. doi: 10.5435/JAAOS-20-02-069.

11. Wang SI, Kwon TY, Hwang HP et al. Functional outcomes of Gartland III supracondylar humerus fractures with early neurovascular complications in children. A retrospective observational study. Medicine (Baltimore). 2017 Jun;96(25):e7148. doi: 10.1097/MD.0000000000007148.

12. Hasler CC. Supracondylar fractures of the humerus in children. Eur J Trauma. 2001;27(1):1-15.

13. Zionts LE, Woodson CJ, Manjra $\mathrm{N}$ et al. Time of return of elbow motion after percutaneous pinning of pediatric supracondylar humerus fractures. Clin Orthop Relat Res. 2009 Aug;467(8):200710. doi: 10.1007/s11999-009-0724-y.

14. Rockwood CA, Green DP, Bucholz RW. Rockwood and Green's Fractures in Adults. Lippincott Williams \& Wilkins; 2006.

15. Hassan FO. Hand dominance and gender in forearm fractures in children. Strategies Trauma Limb Reconstr. 2008 Dec;3(3):101103. doi: 10.1007/s11751-008-0048-6.

16. Lerman JA, Sullivan E, Barnes DA et al. The Pediatric Outcomes Data Collection Instrument (PODCI) and functional assessment of patients with unilateral upper extremity deficiencies. $J$ Pediatr Orthop. 2005 May-Jun;25(3):405-407.

17. Badkoobehi $H$, Choi $P D$, Bae DS et al. Management of the pulseless pediatric supracondylar humeral fracture. J Bone Joint Surg Am. 2015 Jun 3;97(11):937-43. doi: 10.2106/JBJS.N.00983. Review.

18. Robertson AK, Snow E, Browne TS et al. Who gets compartment syndrome? A retrospective analysis of the national and local incidence of compartment syndrome in patients with supracondylar humerus fractures. J Pediatr Orthop. 2018 May/ Jun;38(5):e252-e256. doi: 10.1097/BPO.0000000000001144.

19. Sinikumpu JJ, Victorzon S, Pokka T et al. The long-term outcome of childhood supracondylar humeral fractures: A population-based follow up study with a minimum follow up of ten years and normal matched comparisons. Bone Joint J. 2016 Oct;98-B(10):1410-17.

20. Tuomilehto $N$, Kvisaari $R$, Sommarhem $A$ et al. Outcome after pin fixation of supracondylar humerus fractures in children: postoperative radiographic examinations are unnecessary. Acta Orthop. 2017 Feb;88(1):109-15. doi: $10.1080 / 17453674.2016 .1250058$. 